\title{
Isometries of ultrametric normed spaces
}

\author{
Javier Cabello Sánchez ${ }^{1}$ (D) . José Navarro Garmendia ${ }^{1}$
}

Received: 4 June 2021 / Accepted: 23 August 2021 / Published online: 15 September 2021

(c) The Author(s) 2021

\begin{abstract}
We show that the group of isometries of an ultrametric normed space can be seen as a kind of a fractal. Then, we apply this description to study ultrametric counterparts of some classical problems in Archimedean analysis, such as the so called Problème des rotations de Mazur or Tingley's problem. In particular, it turns out that, in contrast with the case of real normed spaces, isometries between ultrametric normed spaces can be very far from being linear.
\end{abstract}

Keywords Mazur-Ulam theorem · Ultrametric normed spaces · Isometries

Mathematics Subject Classification $46 \mathrm{~S} 10 \cdot 26 \mathrm{E} 30$

\section{Introduction}

The study of isometries dates back to their classification in the Euclidean plane as compositions of translations, reflections and rotations, and comprises a huge variety of results, that include one of the most ancient and celebrated results in the theory of Banach spaces: the Mazur-Ulam Theorem, which states that every onto isometry between real Banach spaces is affine.

In [9], there was an attempt to prove an ultrametric version of the Mazur-Ulam Theorem introducing the notion of non-Archimedean strictly convex space. Nevertheless, Professor A. Kubzdela observed some years later that non-Archimedean strictly convex spaces are a rarity (see [8]).

Communicated by Vesko Valov.

Javier Cabello Sánchez

coco@unex.es

José Navarro Garmendia

navarrogarmendia@unex.es

1 Departamento de Matemáticas, Universidad de Extremadura, Avenida de Elvas s/n, 06006 Badajoz, Spain 
Quite recently, the authors of the present work have shown that any attempt to obtain ultrametric versions of the Mazur-Ulam Theorem via strictly convex spaces is doomed to fail (see [4]).

The aim of this paper is to go a little further and analyse to which extent the behaviour of isometries in ultrametric analysis is far from their behaviour in real analysis. Our main result is Theorem 2.3, whose proof is elementary, and that exhibits a fractal structure of the group of isometries of an ultrametric normed space.

As a consequence, we prove in Corollary 3.6 that the only ultrametric normed spaces that possess a Mazur-Ulam-like property are the trivial examples whose bijections are always affine; namely, the one-dimensional normed spaces over $\mathbb{Z} / 2 \mathbb{Z}$ or $\mathbb{Z} / 3 \mathbb{Z}$, and the two-dimensional normed spaces over $\mathbb{Z} / 2 \mathbb{Z}$.

\subsection{Preliminaries}

The first definitions are a commonplace in ultrametric analysis, and we reflect them just for the sake of completeness, see [9].

Definition 1.1 A non-Archimedean (or ultrametric) valued field is a field $\mathbb{K}$ equipped with a function $|\cdot|: \mathbb{K} \rightarrow[0, \infty)$, called a valuation, such that

(i) $|\lambda|=0$ if and only if $\lambda=0$,

(ii) $|\lambda \mu|=|\lambda||\mu|$,

(iii) $|\lambda+\mu| \leq \max \{|\lambda|,|\mu|\}$ for all $\lambda, \mu \in \mathbb{K}$.

Given any field $\mathbb{K}$, setting $|\lambda|=1$ for any non-zero $\lambda \in \mathbb{K}$ and $|0|=0$ defines a non-Archimedean valuation, called the trivial valuation.

Definition 1.2 An ultrametric normed space is a linear space $X$ over a non-Archimedean field $(\mathbb{K},|\cdot|)$ that is endowed with an ultrametric norm; that is to say, endowed with a function $\|\cdot\|: X \rightarrow[0, \infty)$ such that

(iv) $\|x\|=0$ if and only if $x=0$,

(v) $\|\lambda x\|=|\lambda|\|x\|$, for all $\lambda \in \mathbb{K}, x \in X$.

(vi) $\|x+y\| \leq \max \{\|x\|,\|y\|\}$ for all $x, y \in X$.

Ultrametric normed spaces are metric spaces, so that it makes sense to study isometries between (subsets of) them:

Definition 1.3 Let $A \subseteq X$ and $B \subseteq Y$ be subsets of two ultrametric spaces $X, Y$. A map $f: A \rightarrow B$ is an isometry if for any $x, y \in A$,

$$
\|f(y)-f(x)\|=\|y-x\| .
$$

The inversion through the origin, $x \mapsto-x$, is always an onto isometry $X \rightarrow X$, as so they are translations by a vector $x \mapsto x+z$.

Finally, let us also recall the following well-known fact: 
Lemma 1.4 ([11], 8.C) On an ultrametric normed space $X$, every triangle is isosceles in the big, i.e., for any triplet of points $x, y, z \in X$, the inequality $\|x-z\|<\|y-z\|$ implies $\|y-x\|=\|y-z\|$.

\section{Fractality of onto isometries}

Let $X$ be an ultrametric normed space. Let us denote the open ball and the centred sphere of radius $r \in(0, \infty)$ as

$$
B(x, r):=\{y \in X:\|y-x\|<r\} \quad, \quad S_{X}(r):=\{x \in X:\|x\|=r\},
$$

and let us also convene that

$$
B(x, \infty):=X
$$

Proposition 2.1 Let $r \in(0, \infty]$, and consider an arbitrary family:

$$
f_{r^{\prime}}: S_{X}\left(r^{\prime}\right) \rightarrow S_{X}\left(r^{\prime}\right)
$$

of onto isometries on each sphere $S_{X}\left(r^{\prime}\right)$, for $r^{\prime} \in(0, r)$ (we may have $\left.S_{X}\left(r^{\prime}\right)=\emptyset\right)$.

Then, the map:

$$
f: B(0, r) \longrightarrow B(0, r), \quad f(x):=\left\{\begin{array}{cl}
f_{r^{\prime}}(x), & \text { if } x \in S_{X}\left(r^{\prime}\right) \\
0, & \text { if } x=0
\end{array}\right.
$$

is a centred onto isometry (i.e., an onto isometry such that $f(0)=0)$.

Proof Observe that $\|x\|=\|f(x)\|$, for any $x \in B(0, r)$.

Thus, for any pair of points $x, y \in B(0, r)$,

- if $\|x\|=\|y\|=r^{\prime}$, then

$$
\|y-x\|=\left\|f_{r^{\prime}}(y)-f_{r^{\prime}}(x)\right\|=\|f(y)-f(x)\| .
$$

- if $\|x\|<\|y\|$, then $\|f(x)\|<\|f(y)\|$ and

$$
\|f(y)-f(x)\|=\|f(y)\|=\|y\|=\|y-x\| .
$$

- if $\|x\|>\|y\|$, then $\|f(x)\|>\|f(y)\|$ and

$$
\|f(y)-f(x)\|=\|f(x)\|=\|x\|=\|y-x\| .
$$

The following conditions define equivalence relations on $X$, for any radius $r \in(0, \infty]$, 


$$
x \sim_{r} y: \Leftrightarrow\|y-x\|<r .
$$

The equivalence classes for these relations are precisely the open balls $B(x, r)$. Therefore, an ultrametric normed space $X$, as well as any other subset $A \subset X$, canonically decomposes as a disjoint union of open balls of any fixed radius $r$.

In particular, if we abbreviate the quotient space $S_{X}(r) / \sim_{r}$ as $S / \sim_{r}$ and denote

$$
B_{\bar{x}}(r):=B(x, r) \text {, where } x \in X \text { is any representative of } \bar{x} \in S / \sim_{r},
$$

any sphere decomposes as a disjoint union of open balls:

$$
S_{X}(r)=\bigsqcup_{\bar{x} \in S / \sim_{r}} B_{\bar{x}}(r) .
$$

Proposition 2.2 Let $r \in(0, \infty)$ be a positive radius. If $\sigma$ is a permutation of the quotient set $S / \sim_{r}$ and $\left\{\varphi_{\bar{x}}: B_{\bar{x}}(r) \rightarrow B_{\sigma(\bar{x})}(r)\right\}_{\bar{x} \in S / \sim_{r}}$ is an arbitrary family of onto isometries, then the disjoint union of these isometries

$$
S_{X}(r) \stackrel{f}{\longrightarrow} S_{X}(r) \quad, \quad f(x):=\varphi_{\bar{x}}(x),
$$

is an onto isometry of the sphere $S_{X}(r)$.

Proof Any two points of the sphere $x, y \in S_{X}(r)$ satisfy $\|y-x\| \leq r$ (because of Lemma 1.4), so that the distance between points $x, y \in S_{X}(r)$ in different open balls of (2.1) is exactly $r$.

Therefore, for any pair of points $x, y \in S_{X}(r)$,

- if $\|y-x\|<r$, then $\bar{x}=\bar{y}$ and

$$
\|f(y)-f(x)\|=\left\|\varphi_{\bar{x}}(y)-\varphi_{\bar{x}}(x)\right\|=\|y-x\| .
$$

- if $\|y-x\|=r$, then $\bar{x} \neq \bar{y}$. Hence, $\sigma(\bar{x}) \neq \sigma(\bar{y})$, so that $B_{\sigma(\bar{x})}(r) \neq B_{\sigma(\bar{y})}(r)$ and, in particular, $f(x)$ and $f(y)$ lie in different open balls of (2.1); that is to say, $\|f(y)-f(x)\|=r$.

We can summarize these results as follows:

Theorem 2.3 The following statements hold:

1. (Isometries between balls) Let $B_{1}$ and $B_{2}$ be open balls with the same (possibly infinite) radius $r \in(0, \infty]$. A map $f: B_{1} \rightarrow B_{2}$ is an isometry if and only if there exist translations $\tau, \tau^{\prime}$ and a centred isometry $f_{c}: B(0, r) \rightarrow B(0, r)$ such that

$$
f=\tau^{\prime} \circ f_{c} \circ \tau .
$$


2. (Centred isometries between balls) A centred map $f: B(0, r) \rightarrow B(0, r)$, with $r \in(0, \infty]$, is an isometry if and only if

(a) It preserves norms: $\|f(x)\|=\|x\|$, for any $x \in B(0, r)$.

(b) It is an isometry on each sphere:

$f: S_{X}\left(r^{\prime}\right) \rightarrow S_{X}\left(r^{\prime}\right)$ is an isometry, for any $r^{\prime} \in(0, r)$.

3. (Isometries between spheres) A map $f: S_{X}(r) \rightarrow S_{X}(r)$, with $r \in(0, \infty)$, is an onto isometry if and only if there exist a permutation $\sigma$ of the quotient set $S / \sim_{r}$ and onto isometries $\varphi_{\bar{x}}: B_{\bar{x}}(r) \rightarrow B_{\sigma(\bar{x})}(r)$, for $\bar{x} \in S / \sim_{r}$, such that $f$ is the disjoint union of the $\varphi_{\bar{x}}$ :

$$
S_{X}(r)=\bigsqcup_{\bar{x} \in S / \sim_{r}} B_{\bar{x}}(r) \stackrel{f=\sqcup \varphi_{\bar{x}}}{\longrightarrow} \bigsqcup_{\bar{x} \in S / \sim_{r}} B_{\bar{x}}(r)=S_{X}(r) .
$$

Proof For the first statement, assume $f: B_{1} \rightarrow B_{2}$ is an isometry between open balls of the same radius $r \in(0, \infty]$.

For any pair of points $x \in B_{1}$ and $y \in B_{2}$, the translation by the vector $y-x$ is an isometry $\tau_{y-x}: B_{1} \rightarrow B_{2}$ : in fact, for any $z \in B_{1},\left\|\tau_{y-x}(z)-y\right\|=\|z-x\|<r$, so that $\tau_{y-x}(z) \in B_{2}$.

Therefore, if we take any point $x \in B_{1}$, the translations $\tau_{-x}: B_{1} \rightarrow B(0, r)$ and $\tau_{f(x)}: B(0, r) \rightarrow B_{2}$ are well-defined, and $f_{c}$ is the unique isometry that makes the square commutative:

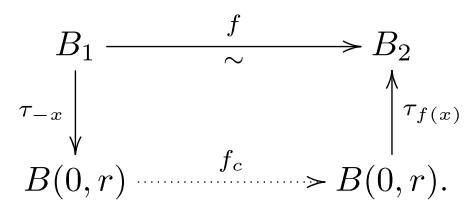

Finally, both the second and third statements are reformulations of Propositions 2.1 and 2.2, respectively.

Example In order to give an explanation of the name of this Section, let us describe the group of centred onto autoisometries of a quite simple space as $X=\mathbb{Q}_{3}^{2}$ when we consider the following valuation and norm:

$$
\left|3^{n} \frac{a}{b}\right|=3^{-n} ; \quad\|(\lambda, \mu)\|=\max \{|\lambda|,|\mu|\},
$$

for every $n, a, b \in \mathbb{Z}$ such that $a$ and $b$ are coprime and $\lambda, \mu \in \mathbb{Q}_{3}$.

The norm $\|\cdot\|$ only takes values in $\{0\} \cup\left\{3^{n}: n \in \mathbb{Z}\right\}$, so the second part of Theorem 2.3 implies that we only need to study the autoisometries $\tau_{n}: S_{X}\left(3^{n}\right) \rightarrow S_{X}\left(3^{n}\right)$ 
for each $n \in \mathbb{Z}$. Later, we can glue these isometries to obtain $\tau: X \rightarrow X$ defined as $\tau(x)=\tau_{n}(x)$ when $\|x\|=3^{n}, \tau(0)=0$.

It is clear that any isometry $\tau_{n}: S_{X}\left(3^{n}\right) \rightarrow S_{X}\left(3^{n}\right)$ is associated with $\tau_{0}: S_{X} \rightarrow S_{X}$ via the dilations $S_{X}\left(3^{n}\right) \rightarrow S_{X}$ and $S_{X} \rightarrow S_{X}\left(3^{n}\right)$, so the structure of Iso ${ }_{X}$ is determined by the structure of Iso $S_{S_{X}}$ : we only need to find one isometry $S_{X} \rightarrow S_{X}$ for each integer $n$ and then glue them all in their corresponding spheres $S_{X}\left(3^{n}\right)$. But we can also take some bijection $\mathbb{Z} \rightarrow \mathbb{N} \cup\{0\}$, like the one defined as $n \mapsto 2 n,-n \mapsto(-2 n-1)$ for every $n \in \mathbb{N}$ and $0 \mapsto 0$. This way, we have a correspondence between Iso $_{X}$ and Iso $_{B_{X}}$ thanks to Iso $_{S_{X}}$. So, let us analyse what happens with Iso $_{S_{X}}$. We can decompose $S_{X}$ as in (2.1), but we have the nice feature that $B_{X}(x, 1)=B_{X}[x, 1 / 3]$ for every $x \in X$ - because $\|y\|<1$ is equivalent to $\|y\| \leq 1 / 3$ - and in particular we get

$$
S_{X}=\bigsqcup_{\bar{x} \in S / \sim_{1}} B_{X}[x, 1 / 3] .
$$

The decomposition given in (2.2) is much more simple than it seems. Namely, as the space that we are dealing with is $X=\mathbb{Q}_{3}^{2}$, the quotient $S / \sim_{1}$ contains only a finite number $k$ of equivalence classes. So, we only need to choose some permutation $\sigma \in S_{k}$ ( $S_{k}$ stands for the symmetric group) and $k$ isometries $\varphi_{i}: B_{X}[0,1 / 3] \rightarrow B_{X}[0,1 / 3]$. Of course, the structure of Iso $_{B_{X}[0,1 / 3]}$ and the one of Iso $_{B_{X}}$ are the same, so we can restrict ourselves to the study of Iso $_{B_{X}} \ldots$

\section{Some consequences}

\subsection{Isotropy of ultrametric spaces}

One of the most famous open problems in functional analysis is the following:

Remark 3.1 (Problème des rotations de Mazur) Let $\left(X,\|\cdot\|_{X}\right)$ be a separable real or complex Banach space and suppose that, for every $x, y \in S_{X}$ there is an onto isometry $f:\left(X,\|\cdot\|_{X}\right) \rightarrow\left(X,\|\cdot\|_{X}\right)$ such that $f(x)=y$. Does this imply that $\left(X,\|\cdot\|_{X}\right)$ is an inner product space, i.e, a Hilbert space? For definitions and results related with Hilbert spaces the reader may see $[6, \S 1]$.

This was solved affirmatively for finite-dimensional spaces and negatively for non-separable spaces, but it is still open in the above form.

In the ultrametric setting, though, the group of isometries always acts transitively on the spheres:

Corollary 3.2 Let $X$ be an ultrametric normed space. For any pair of vectors $x, y \in X$ with the same norm, there exists a centred onto isometry $f: X \rightarrow X$ such that $f(x)=y$. 
Proof For any $x, y \in S_{X}(r)$, there exists an onto isometry $\varphi: S_{X}(r) \rightarrow S_{X}(r)$ such that $\varphi(x)=y$ (Proposition 2.2).

A global isometry is a gluing of isometries on each sphere (Proposition 2.1) so we are done.

Remark 3.3 We distinguish non-Archimedean from ultrametric, although both terminologies are sometimes seen as equivalent. But they do not need to be. Actually, the definition of normed space over a valued field that the reader can find in Bourbaki's monumental work [1] does not involve the ultrametric inequality. Both concepts are equivalent when one refers to valued fields (because a multiplicative norm $|\cdot|: \mathbb{K} \rightarrow[0, \infty)$ satisfies the ultrametric inequality if and only if the image of the natural map $\mathbb{N} \rightarrow \mathbb{K}$ is bounded, [11, 8.2]), but we have found no reason whatsoever to limit the non-Archimedean norms to ultrametric norms.

In fact, recent work [3] points out that it may be interesting to study the structure that usual (not ultrametric) norms give to linear spaces over non-Archimedean fields.

\subsection{Tingley's problem}

Due to the Mazur-Ulam Theorem and Mankiewicz's Theorem, that states that every onto isometry $\tau: B_{X} \rightarrow B_{Y}$ between the unit balls of two real Banach spaces extends to a linear isometry $\tilde{\tau}: X \rightarrow Y$, it stands to reason to wonder whether every onto isometry $\tau: S_{X} \rightarrow S_{Y}$ between the unit spheres of real Banach spaces extends to a linear isometry, too. This is known as Tingley's Problem, and is receiving a lot of attention nowadays, see, e.g., $[5,10]$ and the question can be generalised to does every onto isometry between the boundaries of open convex subsets extend to a linear isometry between the spaces?, see [2]; and to finding minimal requirements on the subset where the isometry is defined, see [7]. One key in this question is that $\tau$ extends linearly if and only if it extends to an isometry, so we have that the question is equivalent, in real Banach spaces, to any of the following:

1. Does every onto isometry $\tau: S_{X} \rightarrow S_{Y}$ extend to an isometry $\tilde{\tau}: X \rightarrow Y$ ?

2. Does every onto isometry $\tau: S_{X} \rightarrow S_{Y}$ extend to a linear map $\tilde{\tau}: X \rightarrow Y$ ?

In ultrametric normed spaces, it is clear that the answer to the first question is yes, with conditions and the answer to the second one is not even close. Please be aware that, for the question to make sense we need to choose a nonempty sphere instead of the unit sphere.

Proposition 3.4 Let $\left(X,\|\cdot\|_{X}\right)$ and $\left(Y,\|\cdot\|_{Y}\right)$ be ultrametric normed spaces over some valued field $\mathbb{K}, r, r^{\prime}>0$ such that $r S_{X} \neq \emptyset$ and $\tau: r S_{X} \rightarrow r^{\prime} S_{Y}$ an onto isometry. If the valuation is not the trivial one, then $r^{\prime}=r$ and $\tau$ extends to an onto isometry $\tilde{\tau}: X \rightarrow Y$. 
Proof Let $x_{0} \in r S_{X}$. The ultrametric inequality implies that $B_{X}\left(x_{0}, r\right) \subseteq S_{X}(0, r)$. It is clear that $\tau: B_{X}\left(x_{0}, r\right) \rightarrow B_{Y}\left(\tau\left(x_{0}\right), r\right)$ is also an onto isometry, so we may compose $\tau$ with translations to get a (centred) isometry $\tilde{\tau}: B_{X}(0, r) \rightarrow B_{Y}(0, r)$ defined as $\tilde{\tau}(x)=\tau\left(x+x_{0}\right)-\tau\left(x_{0}\right)$. Gluing $\tau$ with $\tilde{\tau}$ we obtain an isometry, which we will not rename,

$$
\tilde{\tau}: B_{X}[0, r] \rightarrow B_{Y}[0, r]
$$

Take some $\alpha \in \mathbb{K}$ such that $a=|\alpha|>1-$ it exists because the valuation is not trivial. For any $x \in B_{X}[0, r a] \backslash B_{X}[0, r]$ (equivalently, $r<\|x\|_{X} \leq r a$ ), define $\tilde{\tau}(x)=\alpha \tau(x / \alpha)$. It is clear that $\tilde{\tau}: S_{X}(0, t) \rightarrow S_{Y}(0, t)$ is a well-defined onto isometry for every $t \in(r, r a]$. This implies that $\tilde{\tau}: B_{X}[0, r a] \rightarrow B_{Y}[0, r a]$ is a centred onto isometry. The same way we can define $\tilde{\tau}(x)=\alpha^{n} \tau\left(x / \alpha^{n}\right)$ whenever $x \in B_{X}\left[0, r a^{n}\right] \backslash B_{X}\left[0, r a^{n-1}\right]$, thus finishing the proof.

Remark 3.5 If the valuation of $\mathbb{K}$ is trivial, then we can find at least two pathological behaviours. Namely, if $(\mathbb{Z} / 2 \mathbb{Z})^{2}$ is endowed with the ultrametric norms

$$
\|(a, b)\|_{X}=\max \{|a|, 2|b|\},\|(a, b)\|_{Y}=\max \{|a|, 3|b|\}
$$

then both unit spheres consist in the singleton $\{(1,0)\}$ but the isometry $\tau: S_{X} \rightarrow S_{Y}$ defined as $\tau(1,0)=(1,0)$ does not extend. The same happens if we define the same norm over any other two-dimensional space over a field whose valuation is trivial.

Moreover, if we endow $(\mathbb{Z} / 2 \mathbb{Z})^{2}$ with

$$
\|(a, b)\|_{X}=\max \{|a|, 2|b|\},\|(a, b)\|_{Y}=\max \{2|a|, 3|b|\}
$$

then both $S_{X}$ and $2 S_{Y}$ contain exactly one point. This means that they are trivially isometric, but, with the notations of Proposition 3.4, we have $r^{\prime} \neq r$.

\subsection{Non-existence of Mazur-Ulam type theorems}

Example The map $x \rightarrow-x$ is always a centred isometry $X \rightarrow X$ and, for any fixed $x_{0}$, the map $x \mapsto x+x_{0}$ is an isometry of any sphere $S_{X}(r)$ with $r>\left\|x_{0}\right\|$.

Therefore, for any $r>\left\|x_{0}\right\|$ such that $S_{X}(r) \neq \emptyset$, the maps $f, \widetilde{f}: X \rightarrow X$ defined as

$$
f(x)=\left\{\begin{array}{cl}
-x, & \text { if } x \in S_{X}(r) \\
x, & \text { if } x \notin S_{X}(r)
\end{array}, \quad \widetilde{f}(x)=\left\{\begin{array}{cl}
x+x_{0}, & \text { if } x \in S_{X}(r) \\
x, & \text { if } x \notin S_{X}(r)
\end{array}\right.\right.
$$

are centred onto isometries.

Observe that, whenever they are distinct from the identity, $f$ and $\widetilde{f}$ are non-linear maps.

Example (Isometries of one-dimensional spaces over finite fields) Any finite field $\mathbb{F}_{q}$ is a non-Archimedean field in a unique way: equipped with the trivial valuation $|\lambda|=1$, for any non-zero element $\lambda \in \mathbb{F}_{q}$. As a result, any bijective map $f: \mathbb{F}_{q} \rightarrow \mathbb{F}_{q}$ is an isometry. 
In the same way, every ultrametric norm on a one-dimensional linear space $X_{1}$ over $\mathbb{F}_{q}$ is trivial; i.e., for any such norm there exists $a \in(0, \infty)$ such that $\|x\|=a$ for any non-zero $x \in X_{1}$. Consequently, any bijective map $f: X_{1} \rightarrow X_{1}$ is an isometry.

Corollary 3.6 Let X be an ultrametric normed space. If every centred onto isometry $f: X \rightarrow X$ is a linear map, then one of the following options holds:

1. $X=0$.

2. $X$ is a one-dimensional linear space over $\mathbb{Z} / 2 \mathbb{Z}$.

3. $X$ is a one-dimensional linear space over $\mathbb{Z} / 3 \mathbb{Z}$.

4. $X$ is a two-dimensional linear space over $\mathbb{Z} / 2 \mathbb{Z}$.

Proof If the norm on $X$ is trivial, then any bijection $f: X \rightarrow X$ is an isometry. If $X$ has more than four elements, then we may take different $x_{1}, x_{2} \in X \backslash\{0\}$ such that $x_{3}=x_{1}+x_{2} \neq 0$ and $x_{4} \notin\left\{x_{1}, x_{2}, x_{3}, 0\right\}$ and define $f: X \rightarrow X$ as $f(x)=x$ for every $x \notin\left\{x_{3}, x_{4}\right\}, f\left(x_{3}\right)=x_{4}, f\left(x_{4}\right)=x_{3}$. This is obviously a nonlinear onto isometry from $X$ to $X$.

If $\|\cdot\|$ is not trivial, then we have two options: apart from the value 0 , either $\|\cdot\|$ takes exactly two values or it takes at least three.

If there are $x_{0}, x_{1}, x_{2}$ such that $0<\left\|x_{0}\right\|<\left\|x_{1}\right\|<\left\|x_{2}\right\|$, then we may define the mapping $f: X \rightarrow X$ as

$$
f(x)=\left\{\begin{array}{cl}
x, & \text { if }\|x\| \neq\left\|x_{1}\right\| \\
x+x_{0}, & \text { if }\|x\|=\left\|x_{1}\right\|
\end{array} .\right.
$$

Proposition 2.1 implies that $f$ is an onto isometry and it is not linear because $f\left(x_{2}+x_{1}\right)=x_{2}+x_{1}, f\left(x_{2}\right)=x_{2}, f\left(x_{1}\right)=x_{1}+x_{0}$.

The only option left is that $\|\cdot\|$ takes exactly two positive values, say there are $x_{0}, x_{1} \in X$ such that $0<\left\|x_{0}\right\|<\left\|x_{1}\right\|$.

Consider the map

$$
f: X \rightarrow X, \quad f(x):=\left\{\begin{array}{rl}
x, & \text { if }\|x\|=\left\|x_{1}\right\| \\
-x, & \text { if }\|x\|=\left\|x_{0}\right\|
\end{array} .\right.
$$

This map is a bijection such that $f(0)=0$ and it is clear that fulfills the conditions in Proposition 2.1, so it is an onto isometry.

Moreover, this map is not linear:

$$
f\left(x_{1}+x_{0}\right)-f\left(x_{1}\right)-f\left(x_{0}\right)=x_{1}+x_{0}-x_{1}+x_{0}=2 x_{0} \neq 0 \ldots
$$

unless $2=0$ in $\mathbb{K}$. So, the only problem we have right now is that $\mathbb{K}$ has characteristic 2 , its valuation is trivial (if it is not, then the valuation takes infinitely many values, and so does the norm), and the norm takes exactly two positive values.

If $X$ fulfils everything, then take $0<\left\|x_{1}\right\|<\left\|x_{2}\right\|$. As every triangle is isosceles in the big, for every pair $x, y$ such that $\|x\|=\|y\|=\left\|x_{1}\right\|$ we have $\|x+y\| \leq\left\|x_{1}\right\|$. Furthermore, the valuation of $\mathbb{K}$ is trivial, so 


$$
X_{1}=\left\{x \in X:\|x\| \leq\left\|x_{1}\right\|\right\}=\left\{x \in X:\|x\|=\left\|x_{1}\right\|\right\} \cup\{0\}
$$

is closed under addition and scalar multiplication and this means that it is a vector subspace of $X$ with trivial norm, so if it has only affine isometries it must have 4 elements or less. If there is some nonlinear isometry $X_{1} \rightarrow X_{1}$ that sends 0 to 0 then we just need to extend it to $X$ by identity, so our only problem is that $X_{1}$ has at most four elements. In this case, $\mathbb{K}=\mathbb{Z} / 2 \mathbb{Z}$ implies that $X$ is (linearly) isometric to either $\mathbb{Z} / 2 \mathbb{Z}$ or $(\mathbb{Z} / 2 \mathbb{Z})^{2}$ and $\mathbb{K}=\mathbb{F}_{4}$ implies that $X$ is (linearly) isometric to $\mathbb{K}$.

In the first case, it is easy to see that a bijection $f: X \rightarrow X$ is an isometry if and only if $f\left(x+x_{1}\right)=f(x)+x_{1}$ for every $x \in X$. As the dimension of $X$ is at least 3 , there are $x, y, z \notin X_{1}$ such that $z=x+y$. Now, the map $f: X \rightarrow X$ defined as $f(x)=x$ whenever $x \notin\left\{z, z+x_{1}\right\}, f(z)=z+x_{1}, f\left(z+x_{1}\right)=z$ is an isometry and it is not affine.

In the second case, we only need to consider some linear isometry $\tau: X_{1} \rightarrow X_{1}$ that does not send $x_{1}$ to $x_{1}$-it exists because $X_{1}$ contains four elements- and define $f: X \rightarrow X$ as

$$
f(x)=\left\{\begin{array}{cl}
x & \text { if } x \notin X_{1} \\
\tau(x) & \text { if } x \in X_{1}
\end{array} .\right.
$$

This is an isometry by Proposition 2.1. Furthermore it is not linear because $f\left(x_{2}+x_{1}\right)=x_{2}+x_{1}, f\left(x_{1}\right)=\tau\left(x_{1}\right) \neq x_{1}$ and $f\left(x_{2}\right)=x_{2}$.

To finish the proof, we must observe that if we consider the four element field $\mathbb{F}_{4}$, that can be seen as $\mathbb{F}_{4}=((\mathbb{Z} / 2 \mathbb{Z})[x]) /\left(x^{2}+x+1\right)$, then every centred bijection $\mathbb{F}_{4} \rightarrow \mathbb{F}_{4}$ is an additive isometry. But it is clear that the map $f: \mathbb{F}_{4} \rightarrow \mathbb{F}_{4}$ defined as

$$
f([0])=[0], f([1])=[1], f([x])=\left[x^{2}\right], f\left(\left[x^{2}\right]\right)=[x]
$$

is not $\left(\mathbb{F}_{4}\right)$-linear.

Acknowledgements Supported in part by DGICYT project PID2019-103961GB-C21 (Spain), ERDF and Junta de Extremadura - Consejería de Economía, Ciencia y Agenda Digital programs IB18087, IB20038, GR18001 and GR15152.

Funding Open Access funding provided thanks to the CRUE-CSIC agreement with Springer Nature.

Open Access This article is licensed under a Creative Commons Attribution 4.0 International License, which permits use, sharing, adaptation, distribution and reproduction in any medium or format, as long as you give appropriate credit to the original author(s) and the source, provide a link to the Creative Commons licence, and indicate if changes were made. The images or other third party material in this article are included in the article's Creative Commons licence, unless indicated otherwise in a credit line to the material. If material is not included in the article's Creative Commons licence and your intended use is not permitted by statutory regulation or exceeds the permitted use, you will need to obtain permission directly from the copyright holder. To view a copy of this licence, visit http://creativecommons.org/licen ses/by/4.0/. 


\section{References}

1. Bourbaki, N.: Espaces vectoriels topologiques sur un corps valué, pp. 1-29. Springer, Berlin (2007)

2. Cabello Sánchez, J.: Linearity of isometries between convex Jordan curves. Linear Algebra Appl. 621, 1-17 (2021)

3. Cabello Sánchez, J., Carmona Fuertes, F.: On the concept of non-ultrametric non-Archimedean analysis. Preprint. arXiv:2108.12400 (2021)

4. Cabello Sánchez, J., Navarro Garmendia, J.: A precision on the concept of strict convexity in nonArchimedean analysis. Nonlinear Anal. 207, 112304 (2021)

5. Cabello Sánchez, J.: A reflection on Tingley’s problem and some applications. J. Math. Anal. Appl. 476(2), 319-336 (2019)

6. Conway, J.B.: A Course in Functional Analysis, vol. 96. Springer, New York (2019)

7. Cueto-Avellaneda, M., Peralta, A.M.: Can one identify two unital JB*-algebras by the metric spaces determined by their sets of unitaries? arXiv:2005.04794 (2021)

8. Kubzdela, A.: Isometries, Mazur-Ulam theorem and Aleksandrov problem for non-Archimedean normed spaces. Nonlinear Anal. Theory Methods Appl. 75(4), 2060-2068 (2012)

9. Moslehian, M.S., Sadeghi, G.A.: Mazur-Ulam theorem in non-Archimedean normed spaces. Nonlinear Anal. 69(10), 3405-3408 (2008)

10. Peralta, A.M.: A survey on Tingley's problem for operator algebras. Acta Sci. Math. 84(1-2), 81-123 (2018)

11. Schikhoff, W.: Ultrametric Calculus, an Introduction to p-Adic Analysis. Cambridge Studies in Advanced Mathematics, Cambridge (1984) 